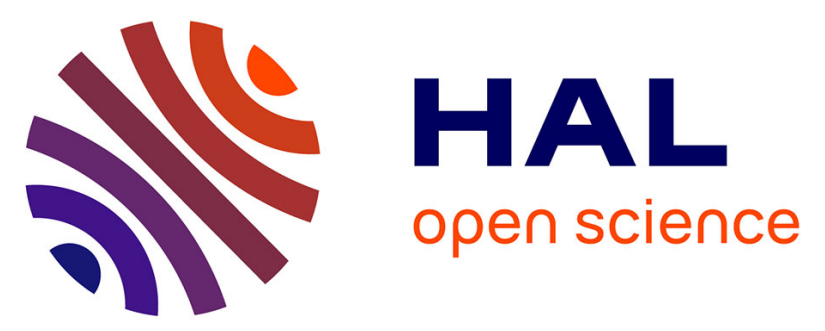

\title{
Évolution du nombre de cas incidents, du stade et des premiers traitements des cancers de la prostate en France entre 2001 et 2016. À partir de données hospitalières de 3 centres \\ G. Delporte, J. Olivier, A. Ruffion, S. Crouzet, C. Cavillon, O. Helfrich, X. Leroy, A. Villers
}

\section{To cite this version:}

G. Delporte, J. Olivier, A. Ruffion, S. Crouzet, C. Cavillon, et al.. Évolution du nombre de cas incidents, du stade et des premiers traitements des cancers de la prostate en France entre 2001 et 2016. À partir de données hospitalières de 3 centres. Progrès en Urologie, 2019, 29, pp.108 - 115. 10.1016/j.purol.2018.12.005 . hal-03486240

\author{
HAL Id: hal-03486240 \\ https://hal.science/hal-03486240
}

Submitted on 20 Dec 2021

HAL is a multi-disciplinary open access archive for the deposit and dissemination of scientific research documents, whether they are published or not. The documents may come from teaching and research institutions in France or abroad, or from public or private research centers.
L'archive ouverte pluridisciplinaire HAL, est destinée au dépôt et à la diffusion de documents scientifiques de niveau recherche, publiés ou non, émanant des établissements d'enseignement et de recherche français ou étrangers, des laboratoires publics ou privés.

\section{(c) (1) $\$$}

Distributed under a Creative Commons Attribution - NonCommerciall 4.0 International 


\section{Évolution du nombre de cas incidents, du stade et des premiers traitements des cancer de la prostate en France entre 2001 et 2016. A partir de données hospitalières de 3 centres}

Gauthier DELPORTE (1), Jonathan OLIVIER (1), Alain RUFFION (2), Sébastien CROUZET (3), Christophe CAVILLON (4), Olivier HELFRICH (1) Xavier Leroy (5) et Arnauld VILLERS

(1) Service d'Urologie CHU Lille, Université de Lille, F-59000 Lille, France

(2) Service d'Urologie Centre hospitalier Lyon sud, Hospices civiles de Lyon, Lyon, France

(3) Service d'Urologie Hôpital Edouard Herriot, Hospices civiles de Lyon, Lyon, France

(4) Service d'Urologie Polyclinique Saint-François - Saint-Antoine, Montluçon, France

(5) Service d'Anatomo-Pathologie CHU Lille, Université de Lille, F-59000 Lille, France

\section{Auteur correspondant}

Gauthier Delporte, Service d'Urologie Hôpital Huriez, CHU Lille, 59037 Lille, France. Tel.: +33 3204442 35; Fax: +33 3204469 15; E-mail: gauthier.delporte@hotmail.fr

\section{MOT CLES (MeSH terms):}

Prostate neoplasms, stage, treatment options, epidemiology, screening

Cancer de prostate, stade, épidémiologie, options de traitement, dépistage, 


\section{Introduction :}

L'INVS a publié en 2018 la mise à jour de l'incidence et de la mortalité française du cancer prostatique jusqu'en 2013 [1]. L'incidence du cancer de prostate a fortement augmenté depuis les années 90 jusqu'au milieu des années 2000, puis a régressé par diminution du réservoir des hommes non dépistés. Parallèlement, la mortalité spécifique a diminué de façon constante. La démocratisation du dépistage individuel par dosage du PSA total a ainsi permis un diagnostic plus précoce de la pathologie à un stade pouvant bénéficier d'une prise en charge curative. La baisse de la mortalité est liée d'une part à cette prise en charge des stades localisés et d'autre part à une amélioration des traitements pour les stades localement avancés et métastatiques.

Cependant, les projections d'incidence et de mortalités sont hasardeuses. La controverse autour du dépistage par PSA, alimentée par les résultats contradictoires des essais ERSPC et PLCO publiés en 2009, ont amené en 2012 à une mise à jour des recommandations de I'USPSTF (United States Preventive Services Task Force) aux USA. Ces recommandations étaient de ne plus doser le PSA (grade D) à titre individuel, et proposaient de ne plus informer les patients de cette possibilité [2]. En conséquence, il a été observé une diminution du nombre de cas diagnostiqués et une augmentation du nombre de cas d'emblée localement avancés ou métastatiques $[3,4]$. Ces constatations et la mise en évidence de biais important au sein de l'essai PLCO ont conduit en 2017 à une révision de la prise de position de l'USPSTF en faveur du dépistage individuel (grade C) [5].

En France, les données épidémiologiques d'incidence ont été actualisées jusqu'à l'année 2013. Les variations du nombre de cas incidents et par stade à partir de 5 séries hospitalières concernant la période 2001-2012 a été publiée [6]. Si l'AFU 
recommande le dépistage individuel depuis 2003, les prises de position divergentes des instances américaines de 2012 à 2017 ont pu avoir un impact sur l'épidémiologie nationale du cancer de prostate.

L'objectif principal de notre étude est de décrire l'évolution du nombre de cas incidents de cancer de la prostate diagnostiqués depuis 2001 jusqu'à la fin de l'année 2016 à partir des données hospitalières de 3 centres en France. Les objectifs secondaires sont de décrire le nombre de cas incidents selon le stade au diagnostic (métastatique et non métastatique, niveau de risque de d'Amico, valeur de PSA et groupe ISUP) et de décrire la répartition des différentes options de premier traitement.

\section{Matériel and Méthodes.}

\section{Population d'étude}

II s'agit d'une étude de cohorte multicentrique sur base de données hospitalières de biopsies prostatiques. Le choix des centres a été fait sur la base du volontariat et de l'absence de registre de cancers dans le même territoire en 2001. Les données ont concerné deux services d'urologie d'établissements publiques (centres hospitaliers régionaux universitaires de Lille et de Lyon-Sud) et un établissement privé (polyclinique Saint François à Montluçon). Les séries de biopsies prostatiques réalisées entre le $1^{\mathrm{er}}$ janvier 2001 et le 31 décembre 2016 chez des patients naïfs du diagnostic de cancer de prostate étaient inclues dans l'étude $(n=11491)$. Les biopsies de réévaluation, correspondant aux patients déjà diagnostiqués et adressés pour un second avis ou ayant préalablement bénéficié d'un traitement curatif ou sous surveillance active, constituaient des critères de non inclusion. 
La sélection et l'inclusion des données au sein des bases informatisées était réalisée à partir des comptes rendus anatomopathologiques des biopsies prostatiques (20012016). Les données de codification ADICAP (Association pour le Développement de I'Informatique en Cytologie et en Anatomie Pathologique) des services d'anatomopathologie des 3 centres ont permis de vérifier l'exhaustivité des données. Une déclaration à la Commission Nationale de l'Informatique et des Libertés était obtenue pour constitution des bases de données.

\section{Recueil des données}

Les données recueillies pour chaque série biopsique étaient : âge du patient, date de biopsie, dosage sanguin du PSA pré biopsique et la présence de néoplasie au sein des carottes biopsiques. Pour les biopsies positives, les données étaient complétées par le stade cT de la classification TNM 2010, le score de Gleason exprimé selon le groupe pronostic conformément aux conférences de consensus ISUP 2005 et 2014 $[7,8]$, le niveau de risque de la classification de d'Amico et le statut métastatique (ganglionnaire ou à distance). Le traitement réalisé chez les patients en cas de positivité a été recueilli pour la période de 2007 à 2016. La présence de néoplasie intra-épithéliale (PIN) isolée était considérée comme une biopsie négative. Le statut métastatique et le premier traitement réalisé n'étaient pas disponibles pour le centre de Lyon-Sud. Les données du centre de Montluçon étaient recueillies pour un tiers des patients après tirage au sort.

\section{Biopsies prostatiques}

Les indications de la réalisation de biopsies prostatiques étaient : une anomalie du toucher rectal, une élévation du PSA $>4 \mathrm{ng} / \mathrm{ml}$, une cinétique d'évolution du PSA $>0,75 \mathrm{ng} / \mathrm{ml} / \mathrm{an}$, une IRM prostatique suspecte. Ces indications pouvaient être

pondérées par d'éventuels facteurs de risques individuels (antécédents familiaux, 
groupes ethniques à risque) et par l'âge ou l'état général du patient. Les biopsies prostatiques étaient réalisées conformément aux recommandations du CCAFU [9]. Sous contrôle échographique, 12 carottes biopsiques étaient réalisées de façon standardisée et intéressaient la partie postérieure de la glande. En cas d'anomalie à l'échographie et/ou à l'IRM pré-biopsique, des biopsies ciblées étaient réalisées avec guidage visuel ou à l'aide d'un logiciel de fusion d'image échographie-IRM (VNav, General Electric Company).

\section{Analyse statistique}

Les paramètres qualitatifs étaient décrits par les effectifs et pourcentages. Les paramètres quantitatifs étaient exprimés en termes de médianes et d'intervalles interquartiles.

\section{Résultats.}

Au total, 11491 séries de biopsies prostatiques ont été réalisées et 5927 cancers diagnostiqués dans les 3 centres au cours des 16 années d'observation (Tableau 1 et Figure 1). L'âge médian au moment du diagnostic était de 67 [61-73] années et le PSA médian était de 7,8 [5,5-13]ng/ml. L'évolution du nombre de biopsies prostatiques et du nombre de cas incidents annuels est représentée dans la Figure

2. L'évolution du nombre de cas incidents au sein des 3 centres montre que le nombre de nouveaux cancers qui était de 239 en 2001 a connu un pic en 2006 avec 478 cas avant d'observer une décroissance de 2006 à 2013 puis était stable de 2013 à 2016, année pour laquelle 321 nouveaux cas de cancer prostatique ont été diagnostiqués. Le taux médian de positivité des biopsies prostatiques était de 52 [50$54] \%$ sur la période d'étude. L'évolution au cours des années des proportions de cas incidents en fonction des stades TNM au diagnostic montrait une stabilité de la 
proportion de lésions diagnostiquées au stade métastatique autour de 8 [7-10]\%, des cancers cT1-T2 autour de 91 [90-93]\% et cT3-T4 autour de 8 [7-11]\%. L'évolution des différentes valeurs de PSA et des groupes de grade ISUP (International Society of Urological Pathology) sont montrées dans les Figures 3 et 4 . Les cancers avec PSA $<10 \mathrm{ng} / \mathrm{ml}$ augmentait de $46 \%$ à $75 \%$ en 2010 puis diminuait à $64 \%$ en 2016 . Les cancers de groupe de grade ISUP 1 diminuait de 59\% à 33\% entre 2011 et 2016. L'évolution des niveaux de risque de d'Amico au cours des années était similaire à l'évolution de la répartition des groupes de grades ISUP et mettait en évidence, à partir de l'année 2012 une diminution de la proportion de lésions classées à bas risque, aux dépens des lésions de risques intermédiaires et hauts, passant de $49 \%$ à $29 \%$ pour l'année 2016 . La répartition des options de premier traitement réalisés pour la période 2007-2016 est présentée dans la Figure 5 pour l'ensemble des patients et dans la Figure 6 pour les cancers à bas niveau de risque de d'Amico. La proportion de premier traitement par surveillance active augmentait de 3 à $21 \%$ entre 2007 et 2016 alors que les prostatectomies restaient stables autour de 46 [44-48]\%. La proportion de premier traitement par surveillance active pour les cancers de faible risque augmentait de 5 à $60 \%$ et par chirurgie diminuait de 73 à $33 \%$. Les lésions de risque intermédiaire bénéficiaient d'une prise en charge chirurgicale dans $64[62-66] \%$ des cas et dans 40 [31-48]\% pour les cancers localisés de hauts risques. 


\section{Discussion.}

\section{Évolution du nombre de biopsies et de diagnostics.}

Le nombre de cas incidents augmentait jusqu'à un pic en 2006 avant de diminuer de 2006 à 2013 puis de se stabiliser de 2013 à 2016. À notre connaissance, il n'y a pas d'autre étude similaire de registres hospitaliers s'étant intéressé aux modifications de cas incidents en France du cancer de prostate depuis l'émission des recommandations de I'USPSTF de 2012. Le pic du nombre de nouveaux cas en 2006 suivi d'une phase de diminution est concordant avec les données nationales et internationales connues qui rapportent un pic d'incidence pour l'année 2005 (64 457 nouveaux cas en France) suivi d'une régression jusqu'en 2013 (48 427 nouveaux cas en France). Dans notre série, le nombre de cas incidents se stabilise pour la période 2013-2016. Parallèlement, les données épidémiologiques INVS font état d'une diminution constante de la mortalité du cancer prostatique sur la période 20012013 [1]. Si le pic d'incidence est lié à la diffusion du dépistage par PSA, la diminution d'incidence observée dans la deuxième moitié des années 2000 est attribuée à la diminution du réservoir d'hommes non dépistés.

\section{Répartition des stades au diagnostic.}

L'apparition du dépistage par PSA avait permis dans les années 90 une diminution importante du nombre de patients métastatiques au diagnostic, passant en France de $17 \%$ en 1995 à $10 \%$ en 2001 [10]. De façon concordante, l'essai ERSPC retrouvait une diminution d'environ $30 \%$ des patients métastatiques dans le bras dépisté après 12 ans de suivi $(p=0,001)[11]$ et le taux de patients diagnostiqués au stade métastatique dans les pays n'utilisant pas le dosage du PSA reste 7 à 8 fois 
supérieur [12]. Ces données expliquent la diminution de mortalité par cancer de la prostate. Contrairement aux récentes publications épidémiologiques aux Etats-Unis $[3,4,13]$, notre analyse n'observe pas d'augmentation de la proportion de lésions métastatiques depuis l'année 2012, stable autour de 8 [7-10]\%. Les données recueillies dans notre étude étant uniquement issues des résultats de biopsies prostatiques, une partie des patients métastatiques diagnostiqués par l'histologie de la métastase (par exemple, patients métastatiques osseux nécessitant un acte de neurochirurgie avec analyse anatomo-pathologique d'un fragment osseux) ne sont pas inclus. Cet élément pourrait biaiser la proportion de diagnostics portés au stade métastatique.

La proportion de lésions diagnostiquées avec un PSA $<10 \mathrm{ng} / \mathrm{ml}$ était en augmentation de $46 \%$ en 2001 à $75 \%$ en 2010 , c'est le reflet des pratiques de dépistage biologique en France. Cependant, cette proportion diminuait à $64 \%$ en 2016, en faveur d'une démarche diagnostique recentrée sur les cancers de volume significatifs. L'utilisation de l'IRM pour diminuer l'indication de biopsies notamment itératives en cas de PSA restant suspect explique cette diminution en proportion des stades de faible risque. Une étude française basée sur les données de l'assurance maladie a noté une diminution modérée des prescriptions de PSA dans la population des hommes de plus de 40 ans de 2011 à 2014 mais les pratiques de dépistage, ainsi que le nombre d'hommes pris en charge pour cancer de la prostate nouvellement diagnostiqué remontaient légèrement en 2015. Le nombre d'hommes de 40 ans et plus ayant bénéficié d'un dosage du PSA dans l'année était stable autour de 30\% entre 2009 et 2011 puis diminuait à 28,7\% en 2012, à 26,9\% en 2014 et remontait à $28,9 \%$ en 2015 [14].

Notre cohorte retrouvait depuis 2011 une diminution importante de la proportion de 
cancers de groupe de grade 1 passant de 59 à 33\% des cancers diagnostiqués en 2016. Parallèlement, on notait une augmentation significative des lésions de groupe ISUP 2, 3 et 4 (présence de grade 4 de Gleason), alors qu'une relative stabilité était observée pour les lésions de groupe 5. Ces résultats sont à mettre en relation avec les modifications d'assignation du score histo-pronostique de Gleason publiées en 2014 par l'International Society of Urological Pathology (ISUP). I'ISUP a reclassé certains profils histologiques antérieurement classés grade 3 de Gleason en grade 4 [8]. Ces modifications peuvent, en partie, expliquer la diminution des scores pronostics de groupe 1 à partir de 2014, mais elles pourraient également être liées à une majoration de l'agressivité histologique en réponse à des facteurs environnementaux par exemple. Ainsi, une diminution de la proportion de cancers classés à faible risque de d'Amico est observée passant de 49\% en 2011 à $29 \%$ en 2016. Ces modifications de répartition des stades de d'Amico ont été largement étudiées en Amérique du nord. De façon concordante, des publications internationales retrouvent une augmentation de la proportion de lésions de risque intermédiaire de d’Amico associée à une diminution des faibles risques à partir de l'année 2010 [15-18].

Les données InVS retrouvaient pour l'année 2008, comme dans notre série, une diminution des cas de cancers prostatiques de hauts risques [19]. Un dépistage adapté à un diagnostic plus précoce et personnalisé, comme l'ont proposé en 2016 le CCAFU en France et le NCCN aux Etats-Unis [20] a pour but de maintenir cette orientation de diminution des cas de cancers prostatiques de hauts risques au diagnostic.

\section{Répartition des options de premier traitement.}


Notre série mettait en évidence un pic d'incidence de la prise en charge par prostatectomie totale en 2010. La proportion de patients traités par prostatectomie variait de 42 à $48 \%$ et celle des patients traités par radiothérapie de 12 à $26 \%$ suivant les années. En France, le nombre de prostatectomies totales est en diminution depuis l'année 2008, passant de 24080 à 17807 procédures annuelles en 2013 soit une diminution de $26 \%$ en 5 ans (source: Intuitive Surgical Inc.). Conformément aux recommandations nationales et internationales, la surveillance active initialement décrite en 2001 par Choo et al [21], devenait une option de choix et augmentait dans notre étude de 3 à $21 \%$ entre 2007 et 2016 et de 5 à $60 \%$ pour les lésions de faibles risques. Les critères de surveillance active utilisés varient de façon importante entre les centres, mais ont tendance à s'étendre en particulier aux lésions de groupe de grade 2, et la proportion d'hommes bénéficiant de cette prise en charge devrait encore augmenter dans les prochaines années aux dépens des prises en charge curatives [22,23]. En moyenne, 5\% des patients de risques intermédiaires ont bénéficié d'une prise en charge par surveillance active, la valeur du dosage de PSA $>10 \mathrm{ng} / \mathrm{ml}$ n'étant pas considérée dans le centre de Lille comme un critère d'exclusion. Les données nationales extraites du registre SEER entre l'année 2004 et 2013 confirment cette tendance aux États-Unis, la proportion de lésion localisées bénéficiant d'une prise en charge par surveillance (surveillance active, surveillance abstention et hormonothérapie) passant de 23 à $32 \%$. Ces modifications de traitement se sont faites aux dépends de la radiothérapie, la proportion de lésions bénéficiant d'un traitement chirurgical restant stable au cours du temps [24]. Les même tendances ont pu être rapportées au Japon parmi 7768 patients, avec cependant des proportions de prise en charge par surveillance active moindre (5,2\% entre 2010 et 2012) [15]. Ces chiffres sont le reflet d'une prise en 
charge adaptée au stade et à l'agressivité du cancer prostatique conformément aux recommandations dans le but de lutter contre le «sur traitement ». Ces évolutions des modalités de surveillance permettent de défendre les politiques de dépistage en population asymptomatique. Parallèlement, les prises en charge locales et plus particulièrement chirurgicales des lésions de haut risque augmentaient au cours du temps.

\section{Limites.}

Une des limites de de notre étude est liée au fait que les centres hospitaliers sont non représentatifs de la population générale. Le statut de centre de référence régional de deux des centres favorise un biais de recrutement. Le fait que les patients métastatiques n'ayant pas bénéficié d'un prélèvement histologique d'origine prostatique ne soient pas inclus dans cette série, constitue une deuxième limite à la généralisation des résultats. L'absence de données concernant les traitements proposés au sein du centre de Lyon Sud limite également la portée des résultats. Les résultats peuvent aussi varier en fonction des modifications des conditions de recrutement des patients dans les centres avec le temps (nombre d'urologue dans chaque centre), mais une stabilité du nombre d'urologues a été constatée pendant la période de l'étude.

\section{Conclusions :}

L'évolution du nombre de cas incidents de cancer de la prostate diagnostiqués depuis 2001 jusqu'à la fin de l'année 2016 à partir des données hospitalières de 3 centres en France semble donner des tendances d'évolution plausibles sur la période d'étude, compatibles avec l'évolution rapportée au sein de la population française. Les données de stade à l'incidence et de traitement montrent une baisse 
du sur diagnostic de cancers à faible risque et de sur traitement par augmentation des options de surveillance. 


\section{References.}

[1] Jéhannin-Ligier K, Dantony E, Bossard N, Molinié F, Defossez G, DaubisseMarliac L, Delafosse P, Remontet L, Uhry Z. Projection de l'incidence et de la mortalité par cancer en France métropolitaine en 2017. Rapport technique. Saint-Maurice $\square$ : Santé publique France, 2017. 80 p.

[2] Moyer VA, U.S. Preventive Services Task Force. Screening for prostate cancer: U.S. Preventive Services Task Force recommendation statement. Ann Intern Med 2012;157:120-34.

[3] Gaylis FD, Choi JE, Hamilton Z, Dato P, Cohen E, Calabrese R, et al. Change in prostate cancer presentation coinciding with USPSTF screening recommendations at a community-based urology practice. Urol Oncol 2017;35:663.

[4] Hu JC, Nguyen P, Mao J, Halpern J, Shoag J, Wright JD, et al. Increase in Prostate Cancer Distant Metastases at Diagnosis in the United States. JAMA Oncol 2017;3:705-7.

[5] Bibbins-Domingo K, Grossman DC, Curry SJ. The US Preventive Services Task Force 2017 Draft Recommendation Statement on Screening for Prostate Cancer: An Invitation to Review and Comment. JAMA 2017;317:1949-50.

[6] Helfrich O, Crouzet S, Ruffion A, Houlgatte A, Cavillon C, Gerard C, et al. Evolution of the number of incident cases of prostate cancer in France from 2001 to 2012 from 5 hospital centers. Prog Urol 2015;25:147-56.

[7] Epstein JI, Allsbrook WC, Amin MB, Egevad LL, ISUP Grading Committee. The 2005 International Society of Urological Pathology (ISUP) Consensus Conference on Gleason Grading of Prostatic Carcinoma. Am J Surg Pathol 2005;29:1228-42.

[8] Epstein JI, Egevad L, Amin MB, Delahunt B, Srigley JR, Humphrey PA, et al. The 2014 International Society of Urological Pathology (ISUP) Consensus Conference on Gleason Grading of Prostatic Carcinoma: Definition of Grading Patterns and Proposal for a New Grading System. Am J Surg Pathol 2016;40:244-52.

[9] Ouzzane A, Coloby P, Mignard J-P, Allegre J-P, Soulie M, Rebillard X, et al. Recommendations for best practice for prostate biopsy. Prog Urol 2011;21:1828.

[10] Jegu J, Tretarre B, Velten M, Guizard A-V, Danzon A, Buemi A, et al. Prostate cancer management and factors associated with radical prostatectomy in France in 2001. Prog Urol 2010;20:56-64.

[11] Schröder FH, Hugosson J, Carlsson S, Tammela T, Määttänen L, Auvinen A, et al. Screening for prostate cancer decreases the risk of developing metastatic disease: findings from the European Randomized Study of Screening for Prostate Cancer (ERSPC). Eur Urol 2012;62:745-52.

[12] Tengue K, Kpatcha TM, Botcho G, Leloua E, Amavi AK, Sikpa K, et al. Profil épidémiologique, diagnostique, thérapeutique et évolutif du cancer de la prostate au Togo. Afr J Urol 2016;22:76-82. 
[13] Weiner AB, Matulewicz RS, Eggener SE, Schaeffer EM. Increasing incidence of metastatic prostate cancer in the United States (2004-2013). Prostate Cancer Prostatic Dis 2016;19:395-7.

[14] Tuppin P, Leboucher C, Dougé M, Peyre-Lanquar G, Gabach P, Descotes JL et al. Dépistage individuel du cancer de la prostate chez les hommes de 40 ans et plus, France, 2009-2015. Données du système national d'information interrégimes de l'Assurance maladie. Bull Epidemiol Hebd 2016;(39-40):700-6.

[15] Tanaka N, Nakai Y, Miyake M, Anai S, Inoue T, Fujii T, et al. Trends in risk classification and primary therapy of Japanese patients with prostate cancer in Nara urological research and treatment group (NURTG) - comparison between 2004-2006, 2007-2009, and 2010-2012. BMC Cancer 2017;17:616.

[16] Hall MD, Schultheiss TE, Farino G, Wong JYC. Increase in higher risk prostate cancer cases following new screening recommendation by the US Preventive Services Task Force (USPSTF). J Clin Oncol 2015;33:143-143.

[17] Dalela D, Loeppenberg B, Sood A, Sammon J, Karabon P, Sun M, et al. MP3914 impact of the 2012 united states preventive services task force recommendation against prostate specific antigen screening on prostate cancer risk group stratification. J Urol 2016;195:e546-7.

[18] Algotar AM, Singh P, Billins J, Thomazin G. Change in prostate biopsy outcomes and costs of care for prostate cancer in underserved population after changes in USPSTF guidelines. J Clin Oncol 2016;34:1548-1548.

[19] Tuppin P, Leboucher C, Samson S, Peyre-Lanquar G, Gabach P, Rebillard X. Vers une évolution des pratiques de détection et de prise en charge du cancer de la prostate chez les hommes de 40 ans et plus en france (2009-2014). Bull Epidemiol Hebd 2016:156-63.

[20] Carroll PR, Parsons JK, Andriole G, Bahnson RR, Castle EP, Catalona WJ, et al. NCCN Guidelines Insights: Prostate Cancer Early Detection, Version 2.2016. J Natl Compr Cancer Netw JNCCN 2016;14:509-19.

[21] Choo R, DeBoer G, Klotz L, Danjoux C, Morton GC, Rakovitch E, et al. PSA doubling time of prostate carcinoma managed with watchful observation alone. Int J Radiat Oncol Biol Phys 2001;50:615-20.

[22] Mohler JL, Armstrong AJ, Bahnson RR, D'Amico AV, Davis BJ, Eastham JA, et al. Prostate Cancer, Version 1.2016. J Natl Compr Cancer Netw JNCCN 2016;14:19-30.

[23] Ploussard G, Isbarn H, Briganti A, Sooriakumaran P, Surcel Cl, Salomon L, et al. Can we expand active surveillance criteria to include biopsy Gleason 3+4 prostate cancer? A multi-institutional study of 2,323 patients. Urol Oncol 2015;33:71.e1-9.

[24] Chen J, Oromendia C, Halpern JA, Ballman KV. National trends in management of localized prostate cancer: A population based analysis 2004-2013. The Prostate 2018;78:512-20. 


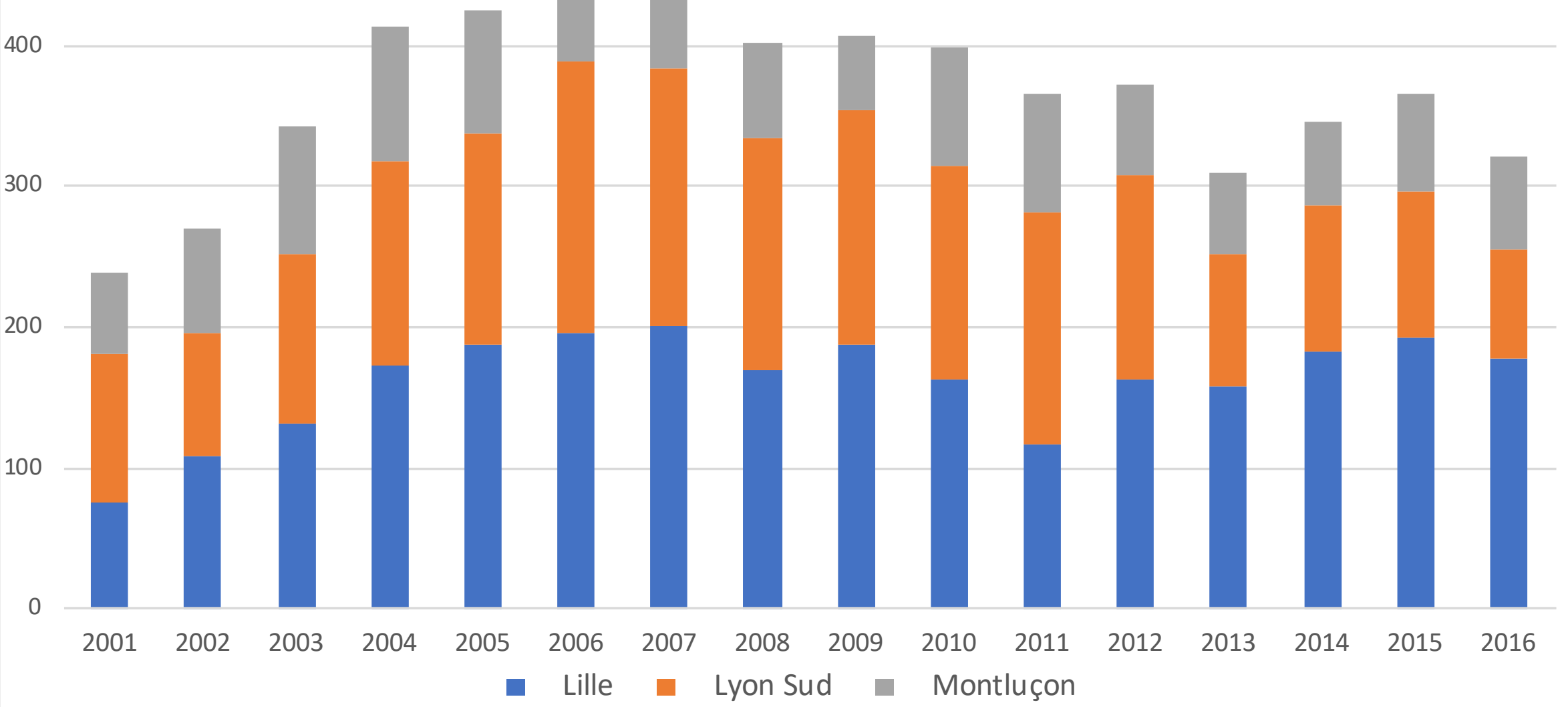


USPSTF

1000

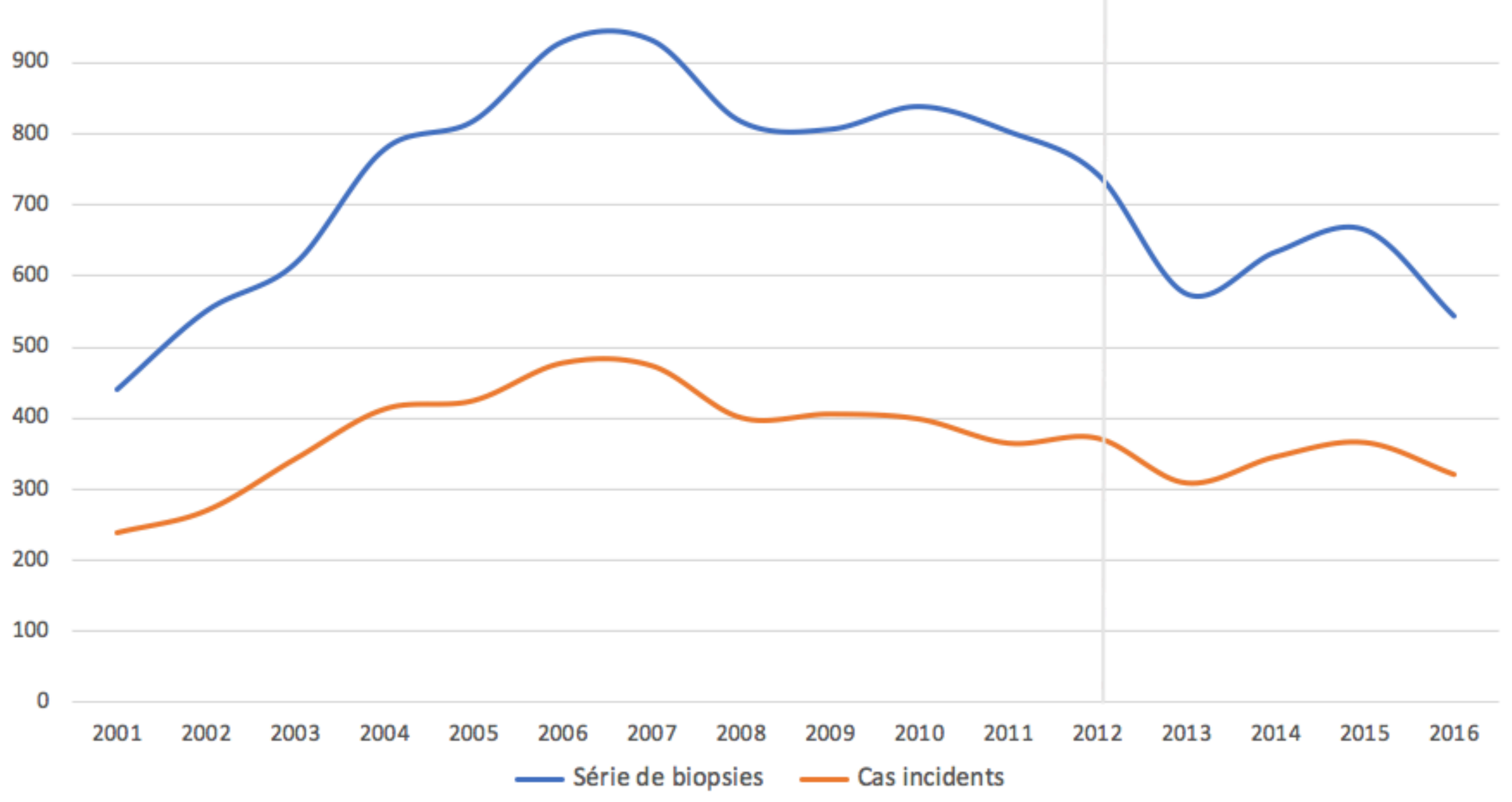


$70 \%$

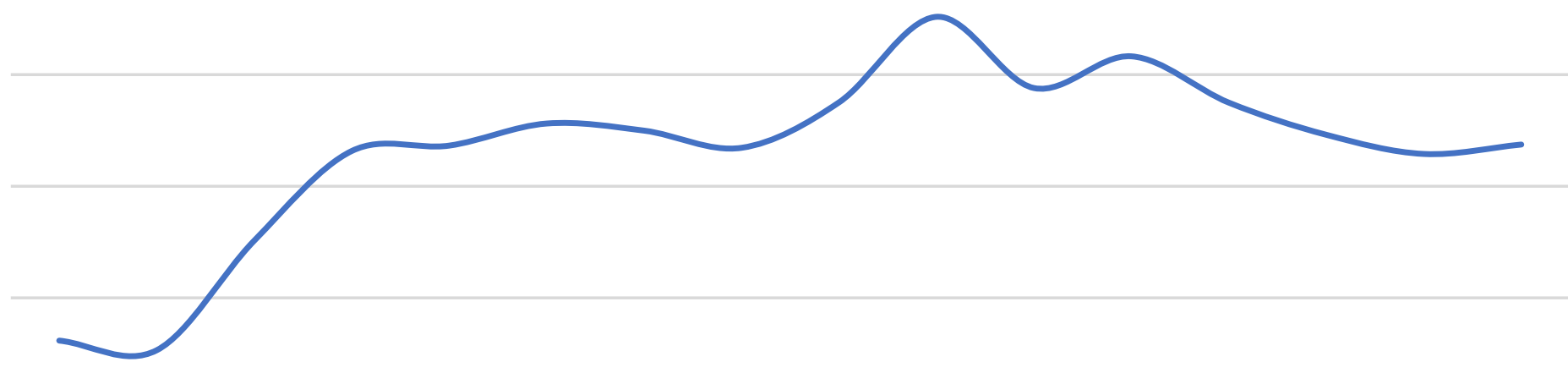

$40 \%$

$30 \%$

$20 \%$

$10 \%$

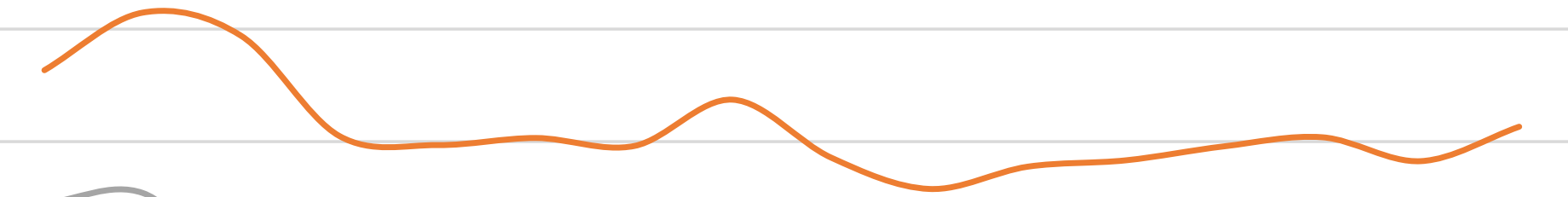

$0 \%$

2001200220032004200520062007200820092010201120122013201420152016 $\longrightarrow<10 \mathrm{ng} / \mathrm{m}$ 10 à $20 \mathrm{ng} / \mathrm{ml}$ 20 à $50 \mathrm{ng} / \mathrm{ml}$ $\longrightarrow 50 \mathrm{ng} / \mathrm{ml}$ 
$50 \%$

$40 \%$

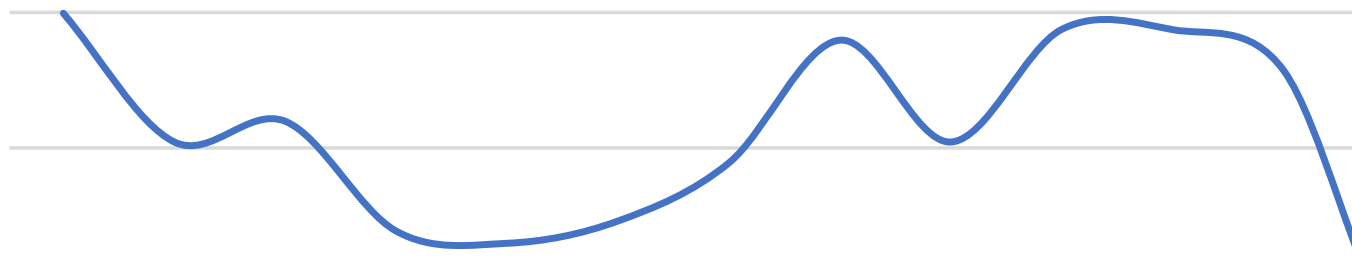

$30 \%$

$20 \%$

$10 \%$

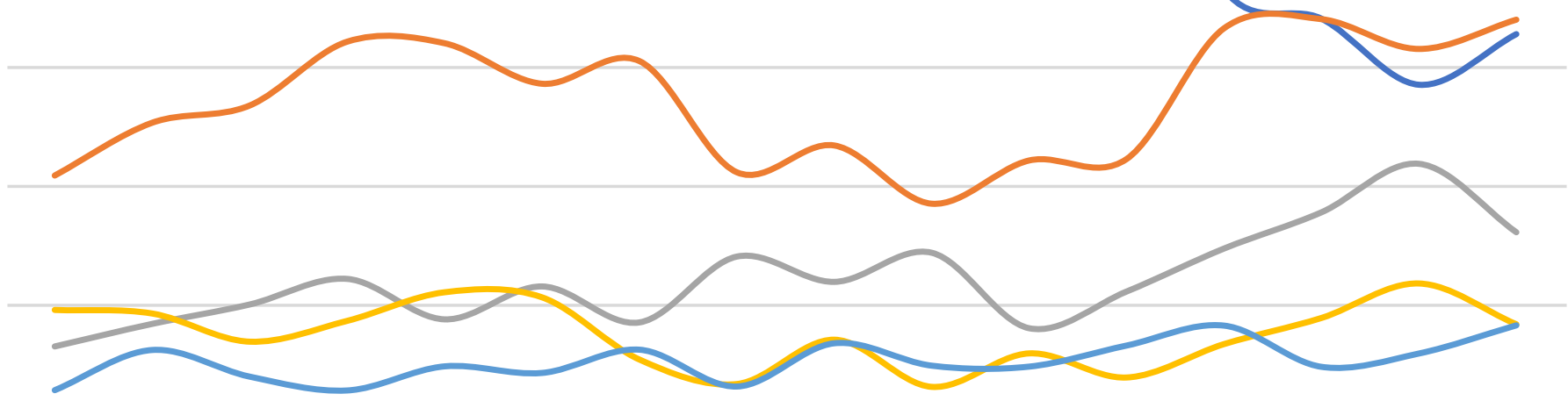

$\begin{array}{llllllllllllllll}2001 & 2002 & 2003 & 2004 & 2005 & 2006 & 2007 & 2008 & 2009 & 2010 & 2011 & 2012 & 2013 & 2014 & 2015 & 2016\end{array}$ Groupe 1

Groupe $2 \longrightarrow$ Groupe 3 - Groupe 4 Groupe 5 

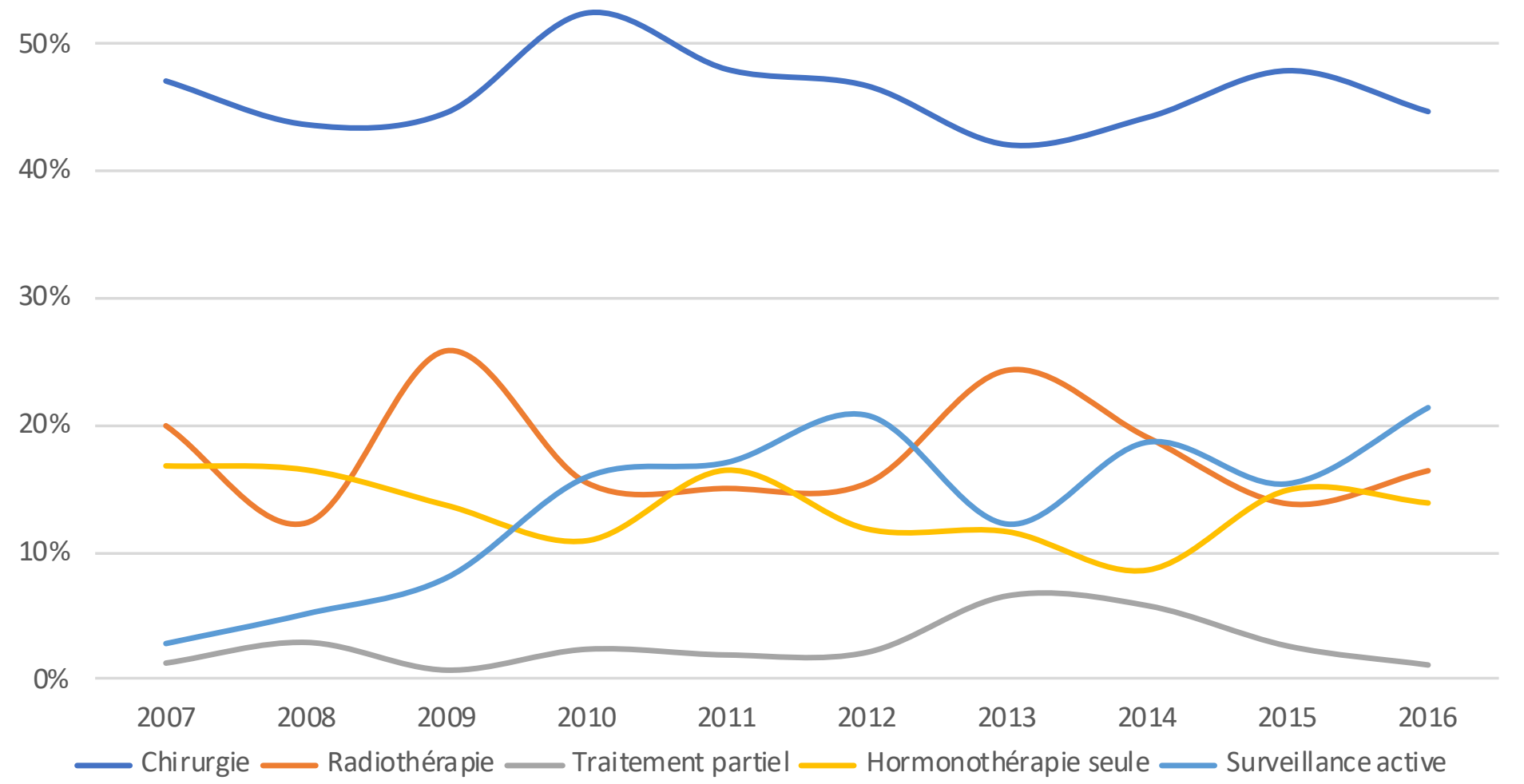


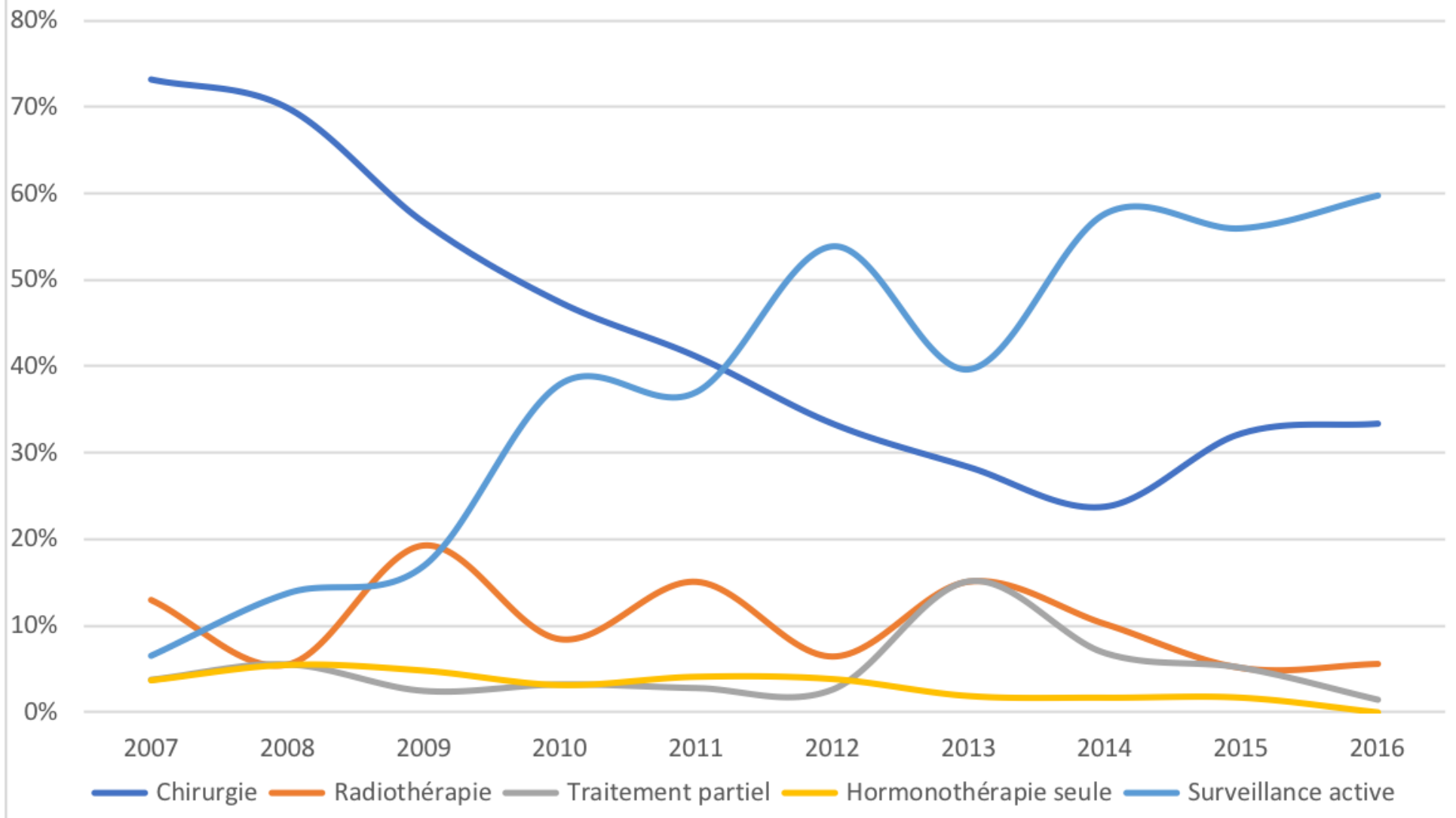




\begin{tabular}{|c|c|c|c|c|c|c|c|c|c|c|c|c|c|c|c|c|c|}
\hline Centre & 2001 & 2002 & 2003 & 2004 & 2005 & 2006 & 2007 & 2008 & 2009 & 2010 & 2011 & 2012 & 2013 & 2014 & 2015 & 2016 & Total \\
\hline \multicolumn{18}{|l|}{ Lille } \\
\hline Nombre de biopsies & 150 & 238 & 224 & 286 & 341 & 371 & 398 & 337 & 327 & 310 & 245 & 267 & 267 & 323 & 344 & 277 & 4705 \\
\hline Nombre de biopsies positives & 76 & 108 & 131 & 173 & 187 & 196 & 201 & 169 & 188 & 162 & 117 & 162 & 157 & 182 & 192 & 178 & 2579 \\
\hline \multicolumn{18}{|l|}{ Lyon-Sud } \\
\hline Nombre de biopsies & 203 & 185 & 240 & 292 & 281 & 362 & 370 & 340 & 347 & 361 & 366 & 337 & 162 & 185 & 184 & 131 & 4346 \\
\hline Nombre de biopsies & 88 & 128 & 154 & 200 & 196 & 196 & 163 & 140 & 132 & 167 & 192 & 139 & 146 & 126 & 137 & 136 & 2440 \\
\hline Nombre de biopsies positives & 58 & 75 & 91 & 95 & 88 & 90 & 90 & 67 & 52 & 84 & 83 & 65 & 57 & 60 & 69 & 66 & 1190 \\
\hline \multicolumn{18}{|l|}{ Total des 3 centres } \\
\hline Nombre de biopsies & 441 & 551 & 618 & 778 & 818 & 929 & 931 & 817 & 806 & 838 & 803 & 743 & 575 & 634 & 665 & 544 & 11491 \\
\hline Nombre de biopsies positives & 239 & 270 & 343 & 413 & 425 & 478 & 474 & 401 & 406 & 399 & 365 & 372 & 309 & 346 & 366 & 321 & 5927 \\
\hline
\end{tabular}

Tableau 1. Nombre de biopsies hors réévaluation et nombre de biopsies positives par centre et par année. 